TITLE:

\title{
Magnetic field stability test of LTS MRI magnet excited by highly stabilized power supply
}

\section{$\operatorname{AUTHOR(S):~}$}

Yoshikawa, M.; Yonemura, N.; Yachida, T.; Imura, T.; Shirai, Y.; Yokoyama, S.

\section{CITATION:}

Yoshikawa, M....[et al]. Magnetic field stability test of LTS MRI magnet excited by highly stabilized power supply. IEEE Transactions on Applied Superconductivity 2016, 26(3): 4401105.

\section{ISSUE DATE:}

2016-04

URL:

http://hdl.handle.net/2433/228376

\section{RIGHT:}

(c) 2016 IEEE. Personal use of this material is permitted. Permission from IEEE must be obtained for all other uses, in any current or future media, including reprinting/republishing this material for advertising or promotional purposes, creating new collective works, for resale or redistribution to servers or lists, or reuse of any copyrighted component of this work in other works.; この論文は出版社版でありません。引用の際には出版社版をご確認ご利用ください。; This is not the published version. Please cite only the published version. 


\title{
Magnetic Field Stability Test of LTS MRI Magnet Excited by Highly-stabilized Power Supply
}

\author{
M. Yoshikawa, N. Yonemura, T. Yachida, T. Imura, Y. Shirai, S. Yokoyama
}

\begin{abstract}
LHe free MRI (HTS-MRI) magnets fabricated by REBCO tape or BSCCO tape superconductors, are expected due to the high-priced LHe. However, HTS-MRI magnet has some problems, stability of magnetic field by use of a power supply in a driving mode and a screening current, for producing a high stability magnet field, both temporally and spatially, necessary for MRI imaging. One solution for these problems is an active magnetic field stability control by use of a power supply instead of the persistent current operation. In this paper, we measured and evaluated the magnetic field stability, using commercial 3TLTS-MRI magnet in the power supply driving mode with the highly-stabilized power supply.
\end{abstract}

Index Terms-LTS-MRI magnet, magnetic field stability, highly-stabilized power supply, persistent current operation

\section{INTRODUCTION}

L TS-MRI magnets of $1.5 \mathrm{~T}$ or $3.0 \mathrm{~T}$ cooled by liquid helium (LHe), whose highly stabilized magnetic field are produced in a persistent current mode with superconducting switches, are widely and commercially applied world-wide.

On the other hand, LHe free MRI (HTS-MRI) magnets fabricated by REBCO tape or BSCCO tape superconductors, are expected due to the high-priced LHe. However HTS-MRI magnet has some problems for producing a high stability magnetic field, both temporally and spatially, necessary for MRI imaging.

One difficulty is the superconducting connection of REBCO tape with extremely low resistivity necessary for the persistent current operation of superconducting MRI magnet. Another important problem is a large screening current [1] induced on the tapes by the self-magnetic field perpendicular to the tapes during the excitation. The screening current induced and decayed on the tapes affects the main magnetic field stability both temporally and spatially.

Automatically generated dates of receipt and acceptance will be placed here; authors do not produce these dates. Acknowledgement of financial support goes here: This research is supported by "Development of Medical Devices and Systems for Advanced Medical Services" from Ministry of Economy, Trade and Industry (METI) and Japan Agency for Medical Research and Development (AMED).

M. Yoshikawa and T. Yachida and Y. Shirai are with Gradiate School of Energy Science, Kyoto University, Kyoto 606-8501, Japan, e-mail: yoshikawa@pe.energy.kyoto-u.ac.jp; $\quad$ yachida@pe.energy.kyoto-u.ac.jp; shirai.yasuyuki.7v@kyoto-u.ac.jp.

N. Yonemura was with Graduate School of Energy Science, Kyoto University, Kyoto 606-8501, Japan, e-mail: yonemura@pe.energy.kyotou.ac.jp.

T. Imura and S. Yokoyama are with Mitsubishi Electric Co, Hyogo , 6618661, Japan, e-mail: Imura.Takeshi@db.MitsubishiElectric.co.jp; Yokoyama.Shoichi@dx.MitsubishiElectric.co.jp.
One solution for these problems is an active magnetic field stability control by use of a power supply instead of the persistent current operation. The important factors for high stability of magnetic field are influence of the stability of the power supply and the screening current. So far, a Bi-2223 HTS MRI magnet has been developed by the group organized by Sumitomo Electric Industries, Ltd and Kobe Steel, Ltd and National Institute for Materials Science and Kyoto University. It could scan images in the power supply driving mode [2]-[4].

In this paper, we measured and evaluated the magnetic field stability, using commercial 3T-LTS-MRI magnet in the power supply driving mode with the highly-stabilized power supply. It is because LTS-MRI magnet has little influence of the screening current. We excited the LTS-MRI magnet of $3.0 \mathrm{~T}$ and scanned MRI images in the power supply driving mode with the highly-stabilized power supply in order to evaluate the magnetic field stability.

\section{EXPERIMENTAL APPARATUS}

\section{A. LTS-MRI magnet of $3.0 \mathrm{~T}$}

Fig. 1 shows LTS-MRI magnet of $3.0 \mathrm{~T}$ provided by Mitsubishi Electric Co.

MRI magnet contains the liquid helium vessel in the vacuum vessel which consists of the superconducting coil, and the persistent current switch (PCS), and the protection diode [5]. MRI magnet equips GM4K refrigerator RDK-408D2 and Compressor Unit F-50H provided by Sumitomo Heavy Industries in order to minimize the evaporation of liquid helium. Inductance of the superconducting coil is $114 \mathrm{H}$.

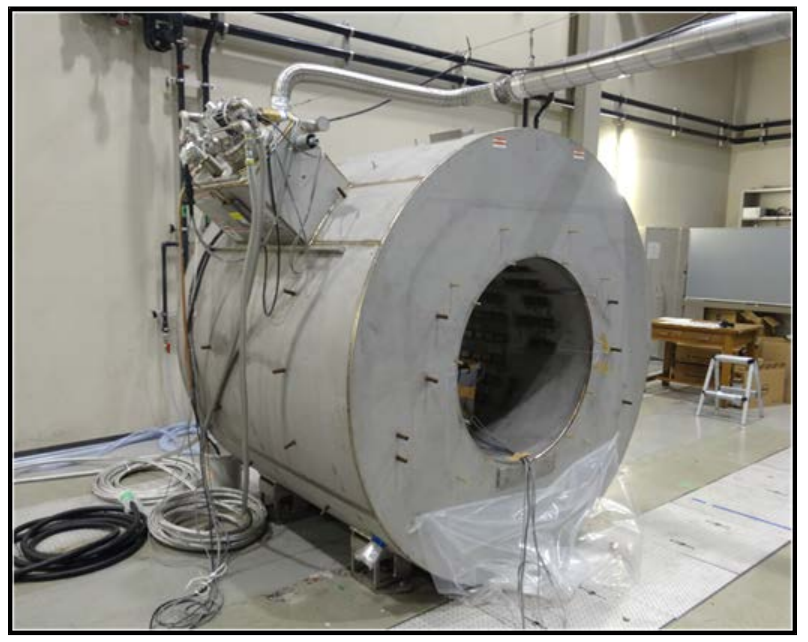

Fig. 1. LTS-MRI magnet of $3.0 \mathrm{~T}$. 


\section{B. NMR probe}

NMR probe can measure the magnetic flux density by relying on the phenomenon of nuclear magnetic resonance. We set up the NMR probe provided by ECHO Electric Co on the center of the MRI coil. The accuracy of the NMR probe is set up $0.1 \mathrm{ppm}$ in this experiment.

\section{Highly-stabilized power supply system}

Fig. 2(a) and Fig. 2(b) show the highly-stabilized power supply unit provided by Kudo Electric Co. The highlystabilized power supply system consists of the highlystabilized power supply and the protective resister and the transformer in Fig. 2(a), and the operation unit and quench detector in Fig. 2(b). This power supply is combined with switching power supply and dropper-type DC stabilized power supply. The control board in the power supply is kept at a constant temperature, therefore output current is highlystabilized and low-noised. Table I shows the power supply specifications.

TABLE I

THE POWER SUPPLY SPECIFICATIONS

\begin{tabular}{cc}
\hline \hline Parameter & Value \\
\hline Output current & $1000 \mathrm{~A}$ \\
Output voltage & $\pm 10 \mathrm{~V}$ \\
Output current stability & $10 \mathrm{ppm} / \mathrm{h}$ \\
\hline \hline
\end{tabular}
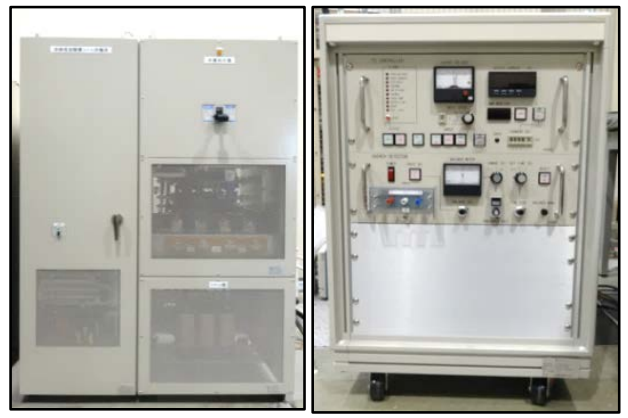

Fig. 2.(a) Highly-stabilized power supply and protective resister and the transformer. Fig. 2 .(b) The operation unit and quench detector.

\section{Current sensor}

Power supply current was measured by using current sensor UP-600A provided by Soft Energy Control Inc. We used VPR221 as a sensing resistor provided by Vishay Intertechnology Inc. Its resistance is $10 \Omega$ and sensitivity is $0.01 \%$. Fig 3 shows the image of the principle of current sensor UP-600A provided by Soft Energy Control Inc [6].

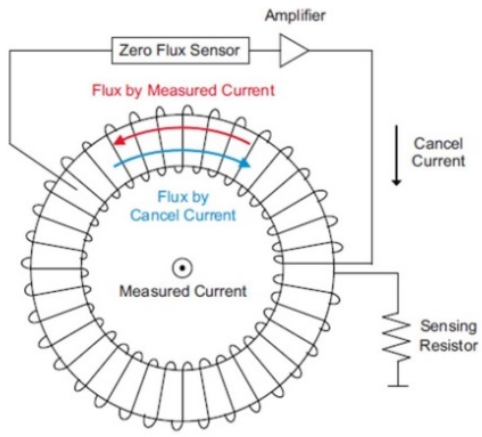

Fig. 3. The principle of current sensor UP-600A provided by Soft Energy
Control Inc [6].

\section{MAgnetic FiELD Stability Test}

\section{A. Experimental Setup}

Fig. 4 shows a circuit diagram of LTS-MRI magnet test. In the power supply driving mode, the PCS (persistent current switch) is opened. On the other hand, in the persistent current mode, the PCS is closed. There are three voltage taps P, C, N on the superconducting coil. The taps $\mathrm{P}$ and $\mathrm{N}$ are on each end and the tap $\mathrm{C}$ is on the center of the coil.

The LTS-MRI coil was excited by highly-stabilized power supply. The current sweep speed is $0.07 \mathrm{~A} / \mathrm{s}$ and the current value was set up $20 \mathrm{~A}, 210 \mathrm{~A}, 237.5 \mathrm{~A}$ in stages. The current value was kept 237.5 A for 2 hours by use of highly-stabilized power supply, and then changed to persistent current operation.

In this experiment, we measured power supply current and voltage difference between the tap $\mathrm{P}$ and $\mathrm{C}$, and magnetic flux density of the center of the coil. Unfortunately, tap voltage $\mathrm{V}_{\mathrm{CN}}$ was missing due to the measuring cable failure.

This experiment is to confirm whether the stability of LTSMRI magnetic field by use of the highly-stabilized power supply is enough for MRI imaging.

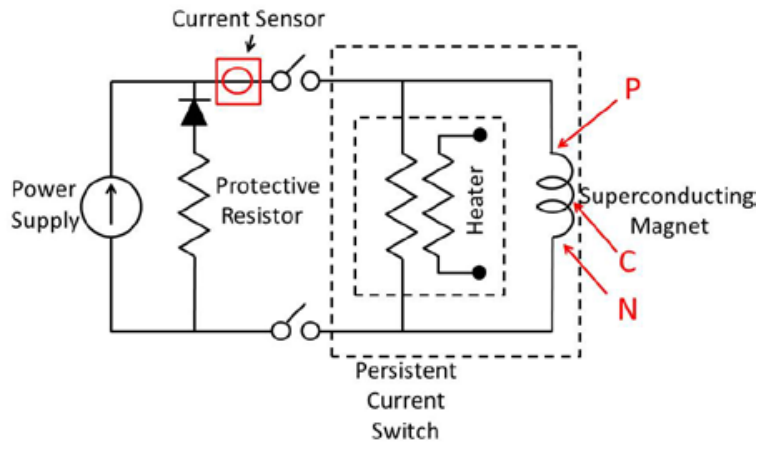

Fig. 4. Circuit diagram of LTS-MRI magnet test.

\section{B. Experimental Result}

Fig. 5 shows current from the power supply, voltage between the tap $\mathrm{P}$ and $\mathrm{C}$ on the MRI coil, magnetic flux density of NMR probe. In this experiment, we started to excite the MRI coil at 15:00 pm.

We evaluated magnetic field stability from 3 to 4 hours after the start of excitation because the voltage across the MRI magnet began stable.

Fig. 6 shows the difference between magnetic flux density $B$ and arithmetic average $B_{\text {ave }}$ calculated during the relevant period. The top shows from 3 to 4 hours by use of power supply and the bottom shows from 6 to 7 hours in the persistent current mode. In each period, the values of magnetic flux density stability

$$
\frac{B_{\text {Max }}-B_{\min }}{B_{\text {ave }}} \times 10^{6}
$$

are $0.755 \mathrm{ppm} /$ hour in the power supply driving mode and $1.07 \mathrm{ppm} /$ hour in the persistent current mode. These stabilities of magnetic field are enough for MRI imaging.

Fig. 7 shows magnetic flux density in the persistent current mode. During 100 to 120 hours after the start of excitation, we obtained high stability magnetic field $0.0503 \mathrm{ppm} /$ hour though it was larger than $1.0 \mathrm{ppm} /$ hour immediately after we changed 
to the persistent current mode. In the persistent mode, magnetic flux density decayed exponentially. This may be due to the coil joint resistance at the persistent switch.
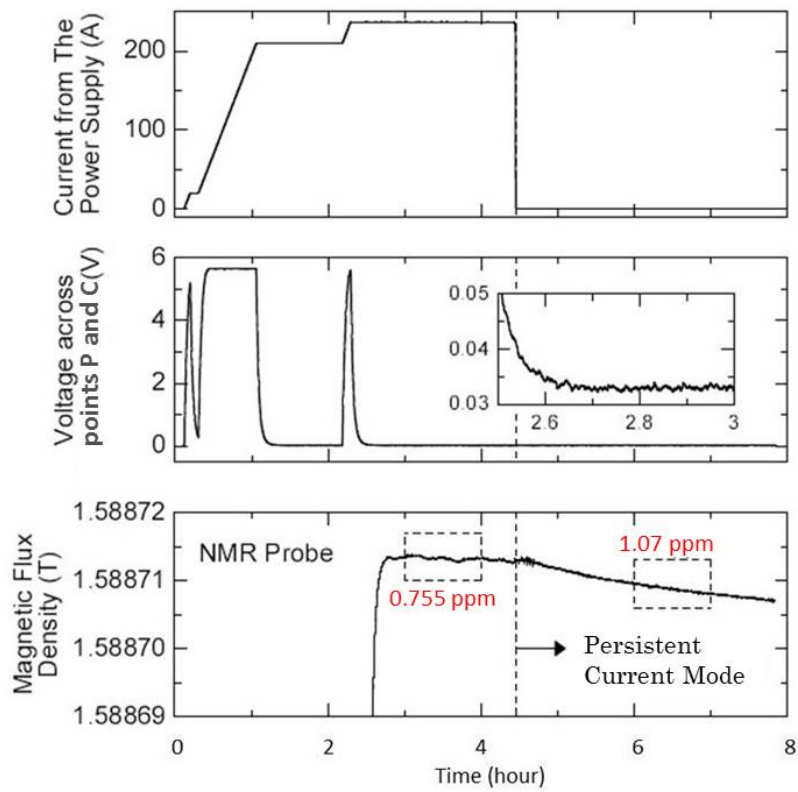

Fig. 5. Current from the power supply, Voltage between the tap $\mathrm{P}$ and $\mathrm{C}$ on the MRI coil, Magnetic flux density of NMR probe.
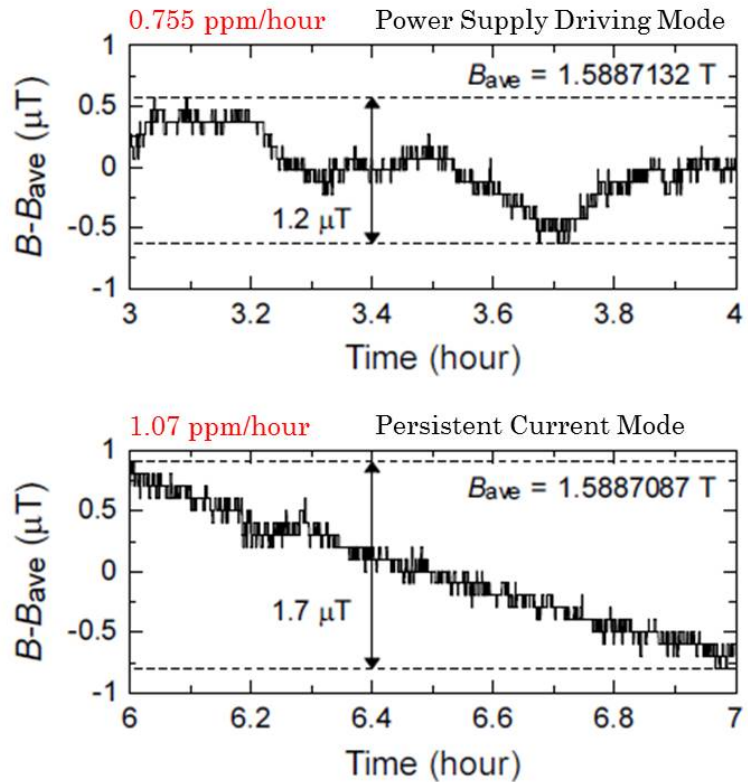

Fig. 6. Difference between magnetic flux density and the average $B_{\text {ave }}$ measured by the NMR probe.

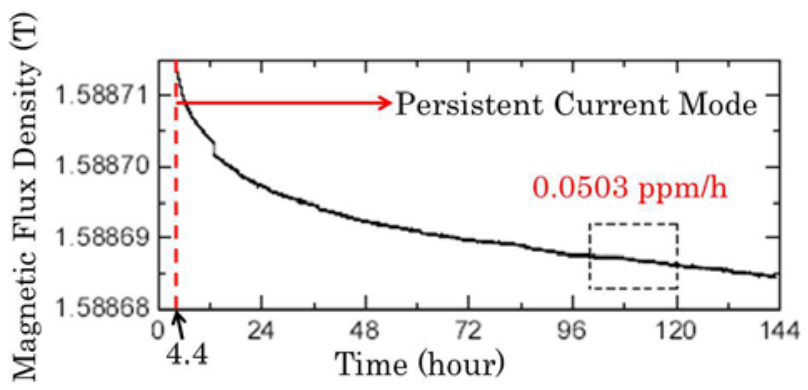

Fig. 7. Magnetic flux density in a persistent current mode.
IV. MRI IMAGING TEST

IN THE POWER SUPPLY DRIVING MODE

\section{A. Experimental Setup}

We excited LTS-MRI coil by the highly-stabilized power supply. The current sweep speed is $0.07 \mathrm{~A} / \mathrm{s}$ and we set up current value 224.3 A. We kept current value 224.3 A for 5 hours by use of highly-stabilized power supply.

In this experiment, we measured power supply current and voltage across the MRI magnet, and magnetic flux density of the center of the coil. Magnetic flux density is measured for 2.75 hours from the start of excitation. After that, we replaced the NMR probe with the MRI imaging apparatuses. Then, we scanned the images of a citrus depressa, a kind of a citrus fruit, whose diameter is $20 \mathrm{~mm}$, under the condition of highlystabilized magnetic field. A MRI imaging sensor detects hydrogen molecules to scan, therefore a juicy citrus depressa is appropriate for MRI imaging.

This experiment is to confirm whether MRI images can be scanned in the condition of the power supply driving mode.

\section{B. Experimental Result}

Fig. 8 shows current from the power supply and voltage between the tap $\mathrm{P}$ and $\mathrm{N}$ on the MRI coil. We set up current value 224.3 A and kept for 5 hours. The current value was kept constant by the control of the highly-stabilized power supply. The power supply reached the target current value and kept constant current after an hour from the start of excitation. The voltage became stable 1.5 hours after the start of excitation. It took half an hour that the voltage became almost constant from the start of constant current control. This may be caused since the power supply can control only the supply current to the MRI circuit which consists of MRI magnet and PCS resistance in parallel.

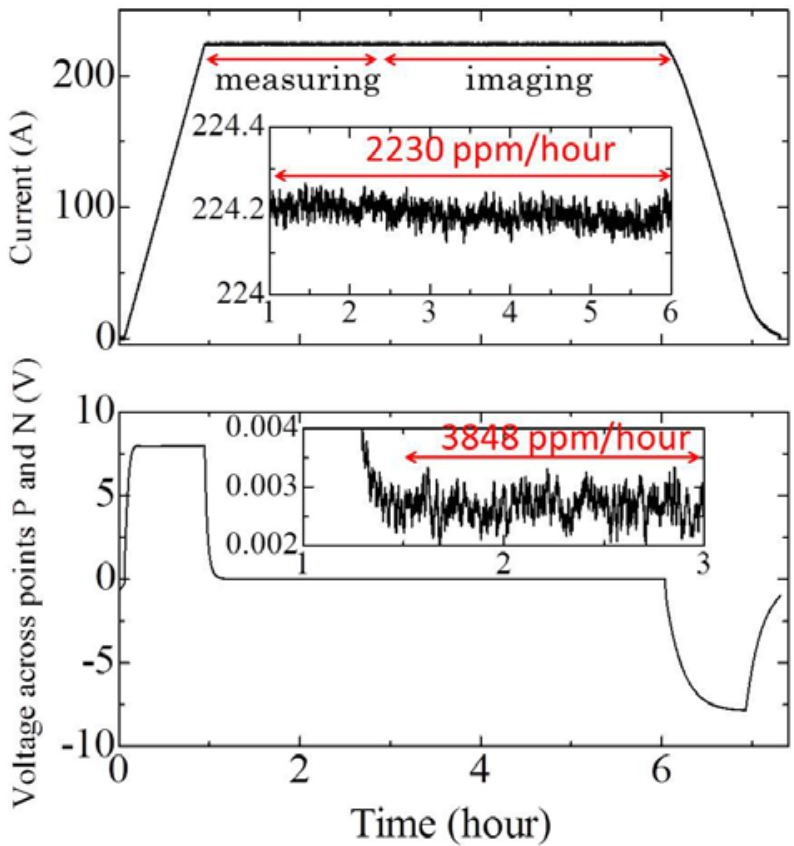

Fig. 8. Current from the power supply and Voltage across the MRI magnet. 
Fig. 9 shows magnetic flux density of NMR in the power supply driving mode. Fig. 10 shows the difference between magnetic flux density $B$ and the average $B_{\text {ave }}$ measured by the NMR probe from 1.5 to 2.75 hours. The stability of magnetic flux density is $0.961 \mathrm{ppm} /$ hour from 1.5 to 2.75 hours. Magnetic flux density decays temporally as well as the case in the persistent current mode. The result is similar to the test of excitation of LTS-MRI magnet in the power supply driving mode and in the persistent current mode under the similar condition. Small disturbance was observed at around 12:00 pm. It may be caused by the power source voltage fluctuation at lunchtime. In the power supply driving mode the stability of magnetic field is affected by the power source voltage fluctuation. We must take it into consideration for high stabilized magnetic field.

Fig. 11 shows MRI images of a citrus depressa, a kind of citrus fruit, whose diameter is $20 \mathrm{~mm}$. The surface of a citrus depressa was scraped to fit the hall size of the MRI imaging apparatus. Imaging was begun after 2.75 hours from the start of excitation under the highly-stabilized magnetic field, less than $1.0 \mathrm{ppm} /$ hour. Clear images were obtained due to the high stability of magnetic field.

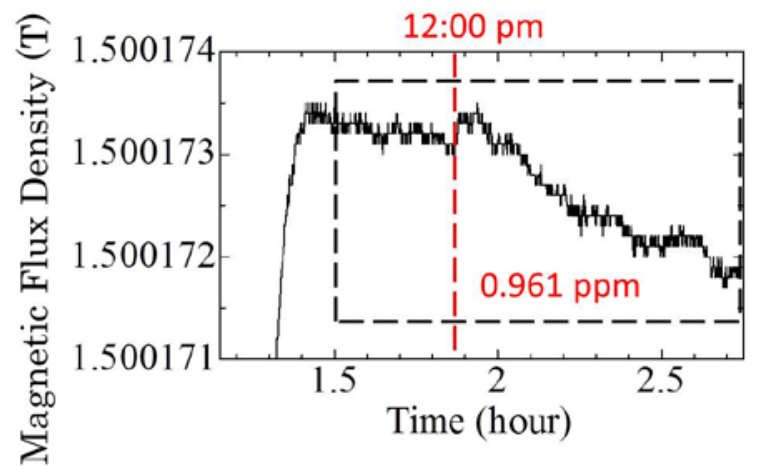

Fig. 9. Magnetic flux density of NMR in the power supply driving mode.

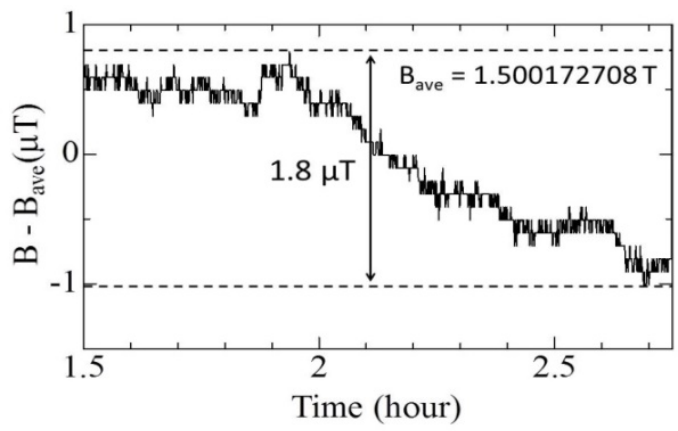

Fig. 10. Difference between magnetic flux density and the average $B_{\text {ave }}$ measured by the NMR probe from 1.5 to 2.75 hours.

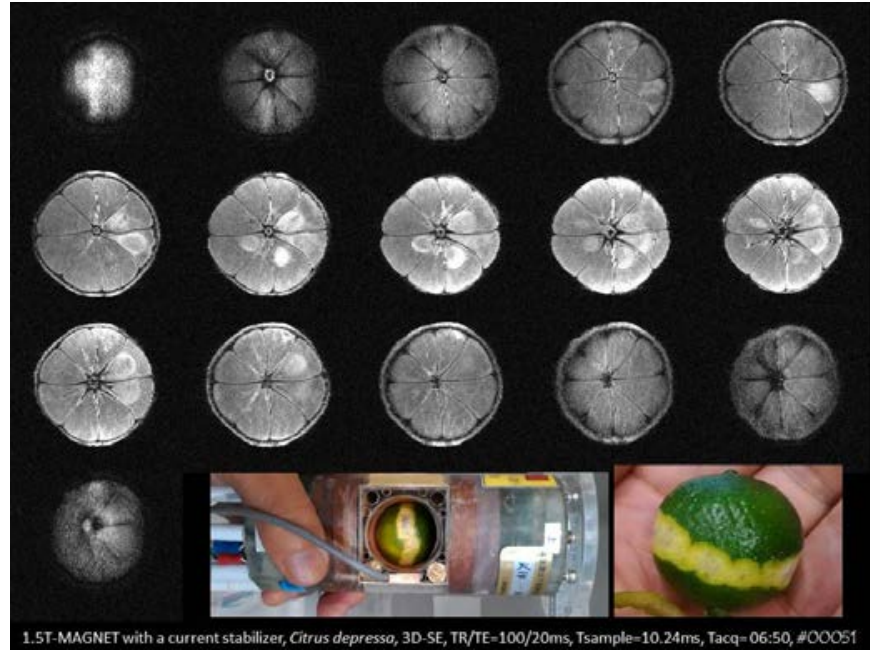

Fig. 11. MRI images of citrus depressa, a kind of citrus fruit.

\section{DISCUSSION}

Our target is to realize a HTS MRI system. HTS MRI magnet cannot be operated in the persistent current mode due to a relatively large wire joint resistance. We must realize a high stabilized magnetic flux density in the power supply driving mode. There is also the screening current problem in the HTS MRI magnet. For the first step, we measured the stability of magnetic flux density of LTS MRI magnet in the power supply driving mode. It is proper to use LTS MRI magnet for evaluating only the influence of the power supply because LTS MRI has little influence of the screening current.

Table II shows comparison with magnetic field stability in the power supply driving mode and the persistent current mode. In the power supply driving mode, the stability of magnetic field is $0.755-0.961 \mathrm{ppm} /$ hour and in the persistent current mode, the stability is $0.0503 \mathrm{ppm} / \mathrm{hour}$. Current is measured by current sensor at output current from the power supply. The control board in the power supply is kept at a constant temperature and output current is accuracy, but the magnetic stability required is beyond the resolution of current sensor. Therefore, we must feed back another physical factor, for example magnetic flux density measured with NMR probe, in order to control magnetic field stability by use of the power supply. On the other hand, in the persistent current mode, the stability of magnetic field is smaller than in the power supply driving mode by one order of magnitude. We try to make a MRI feedback system for more highly-stabilized magnetic field in the future.

TABLE II

COMPARISON WITH MAGNETIC FIELD STABILITY IN THE POWER SUPPLY DRIVING MODE AND THE PERSISTENT CURRENT MODE

\begin{tabular}{cc}
\hline \hline Power supply driving mode & Persistent current mode \\
\hline $0.755-0.961 \mathrm{ppm} / \mathrm{hour}$ & $\begin{array}{c}0.0503 \mathrm{ppm} / \mathrm{hour} \\
\text { (decay exponentially) }\end{array}$ \\
\hline \hline
\end{tabular}




\section{CONCLUSION}

In order to apply a REBCO magnet to MRI system, we must evaluate the effect of not only the screening current but also the power supply current fluctuation upon the magnetic temporal and spatial stability.

For the first step, we operated a 3T-LTS-MRI magnet with the power supply driving mode instead of the persistent current mode. Since the LTS magnet has less screening current, we can evaluate the magnetic fluctuation only due to the power supply.

As a result of experiment, high stability of magnetic field (less than $1 \mathrm{ppm} / \mathrm{h}$ ) was achieved in the power driving mode and clear images $(20 \times 20 \mathrm{~mm})$ were obtained successfully. Thus the stability of the power supply has a small influence on magnetic flux density stability for MRI imaging.

For the next step in near future, we will make an operation test of a HTS-MRI of REBCO tape in the power supply driving mode in order to evaluate the screening current effects on the main magnetic field stability.

\section{REFERENCE}

[1] Y. Yanagisawa and H. Maeda: "Mechanism and Suppressive Methods for Screening-current-induced Magnetic Field of REBCO Coils", Journal of the Cryogenic Society of Japan, 48, 4, pp. 165-171 (2013)

[2] H. Kitaguchi, O. Ozaki, T. Miyazaki, N. Ayai, K. Sato,S. Urayama and H. Fukuyama: "Development of a Bi-2223 HTS Magnet for 3T MRI System for Human Brains", IEEE Transactions on Applied Superconductivity, 20, 3, pp. 710-713 (2010).

[3] S. Urayama, H. Fukuyama, O. Ozaki, H. Kitaguchi, K. Takeda, I. Nakajima, N. Oonishi,M. Poole and K. Sato: "Cryogen-free 3T-MRI system for human hightemperature superconducting tapes", 2012 ICME International Conference on Complex Medical Engineering (CME), pp. 376-381 (2012).

[4] Y. Terao, O. Ozaki, C. Ichihara, S. Kawashima, T. Hase, H. Kitaguchi, S. Kobayashi, K. Sato,I. Nakajima, N. Oonishi, M. Poole, K. Takeda, S. Urayama and H.Fukuyama: "Newly De-signed $3 \mathrm{~T}$ MRI Magnet Wound With Bi-2223 Tape Conductors", IEEE Transactions on Applied Superconductivity, 23, 3, p. 4400904 (2013).

[5] S. Yamamoto: "Use of superconducting technology for medical care”, Electrical Review, 98, 7, pp. 36-39 (2013)

[6] Soft Energy Control Inc: "Current Sensor, Products”. $\quad$ http://www.softenergycontrols.co.jp/product/sensor.html 
